\title{
Paste reticulation blockage: an Australian case study
}

\author{
AS Morcombe Quattro Project Engineering Pty Ltd, Australia
}

\begin{abstract}
This paper looks at the design of a paste reticulation system, particularly a new method of diverting paste flows in critical situations, and the safeguards that were incorporated into the reticulation design to minimise the risk of line blockage during backfill operations. It also details the actions taken during a paste blockage event to flush and recover the entire line.

The paper considers some of the restrictions that were imposed due to cost and time restraints on the schedule, and the impact these restrictions had on the profile and the flow characteristics of the reticulation network. Finally, a summary of key learnings and solutions that have been implemented to further safeguard the system has been provided.
\end{abstract}

Keywords: paste fill, blockage, reticulation, Quattro Diverter 2 position (QD2), Quattro Changeover Spool (QCS), paste flushing stations (PFS)

\section{Introduction}

CSA Mine, owned and operated by Cobar Management Pty Ltd, is based in Cobar, regional New South Wales, Australia. The mine has a newly commissioned paste plant, filter plant and underground reticulation system. The surface-to-underground (9430L) borehole of $835 \mathrm{~m}$ for its entirety consists of an outer casing of $250 \mathrm{~mm}$ and an inner 'replaceable' casing that is $175 \mathrm{~mm}$ in diameter for the first $12 \mathrm{~m}$ and $110 \mathrm{~mm}$ in diameter for the remaining $835 \mathrm{~m}$. The pressure generated within a vertical borehole of this depth would exceed the pressure rating of the underground reticulation rapidly if an engineering solution were not implemented.

The implemented engineering solution is a reliable means of diverting or dumping the contents of the reticulation system via a Quattro Diverter 2-position (QD2). Due to the high pressures, it was decided automated control and underground pressure sensors were to be integrated with the control systems at the paste plant, thus forming the safest and most reliable way to protect the reticulation from blockage and personnel from risk of over-pressurised pipes.

This case study focuses on a blockage event that occurred on 6 July 2018 at about 1,600 m vertical depth from the surface.

\section{Underground reticulation layout}

The underground reticulation system consists of 150 NB schedule 80 steel pipe rated to $10 \mathrm{MPa}$. These 150 NB reticulation lines connect with inter-level boreholes, three of which are cased with a reduced diameter of 110 NB steel pipe. The remainder are unlined drill holes (e.g. approximately $150 \mathrm{~mm}$ ID) with 150 NB steel inserts top and bottom.

The reticulation system is about $1850 \mathrm{~m}$ in total length. The lowest QD2 and permanent flushing head installed at the time of the blockage was at the $8790 \mathrm{~L}$, with the lowest installed reticulation at the $8610 \mathrm{~L}$. Figure 1 presents an overall layout of the underground paste reticulation system identifying key areas and levels. 


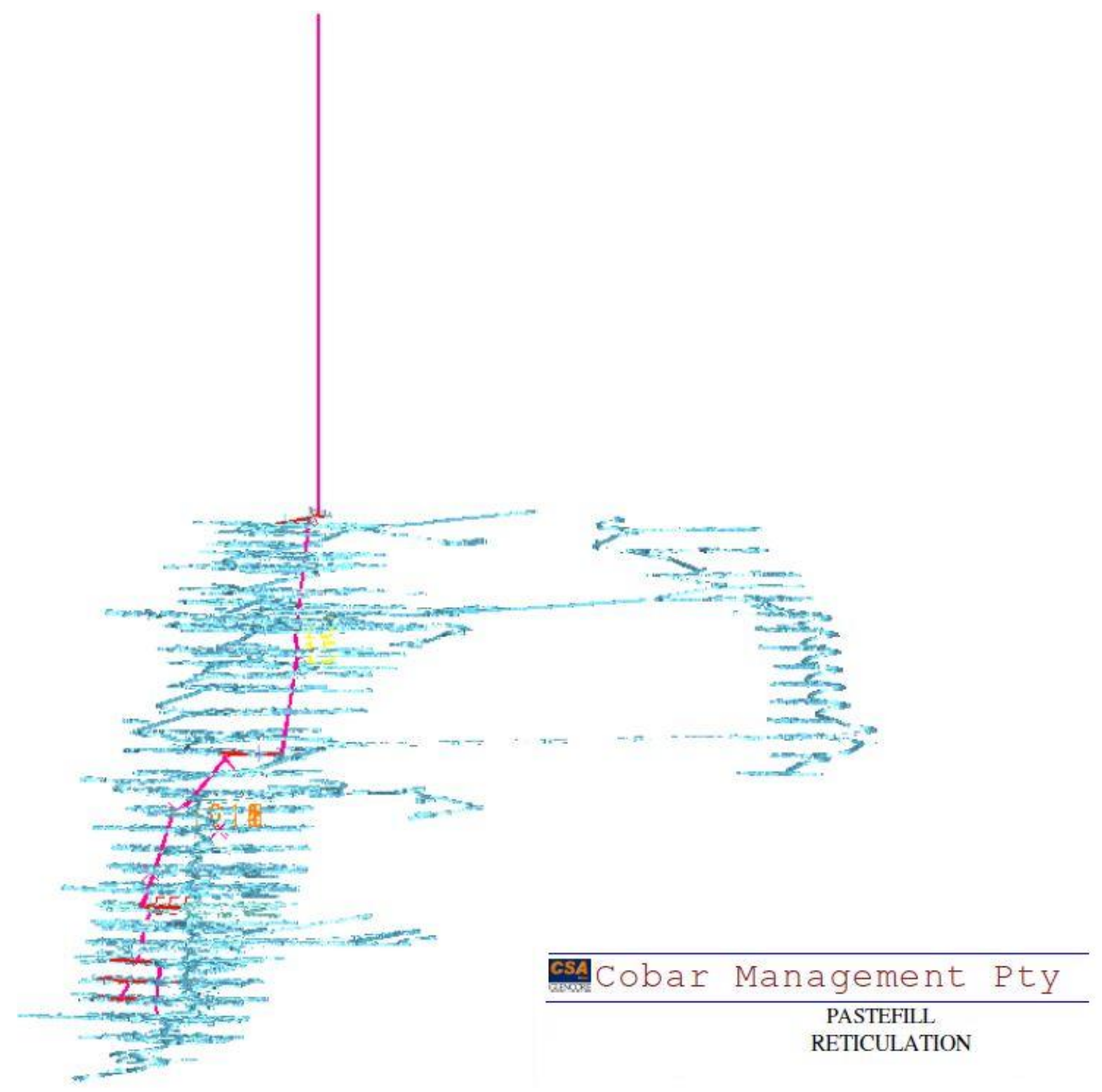

Figure 1 Schematic diagram of the CSA underground paste reticulation system

\section{Installed Quattro Diverter 2 position}

Through extensive flow modelling, the first three QD2s were installed at strategic locations, the first of which was located at the bottom of the surface-to-underground borehole (9430L). Figure 2 shows the installed QD2 at the $9040 \mathrm{~L}$. Paste enters the diverter from the rear and either flows inline or is diverted to a dump location as required during an emergency dump situation.

A Vegabar82 pressure sensor is incorporated into the paste line, reporting back to the control boxes of each QD2 and to the surface paste plant control system to allow real-time pressure monitoring of the paste system and also to initiate an 'emergency dump sequence' if a set maximum pressure is detected. Each QD2 has a closed circuit with a pressure sensor, pressure switch, UPS battery backup system and an individual dedicated air receiver to enable the QD2s to automatically divert the paste stream to a dump location in the event of a system over-pressure situation. Over-pressure can cause consequences such as concurrent power failure, loss of air supply or loss of communication with the paste plant control system (such as a severed fibre optic cable).

One of the most attractive benefits of the automated dump function at the mine is the instant response to a pressure spike; the QD2 can be pre-set to actuate at any predetermined pressure. The QD2s are also fitted 
with a pressure-welded flushing head with non-return valves (to prevent filling the mine services with paste) and were plumbed into the mine's water supply and air supply services at each QD2 location, thus forming an underground flushing system. A mobile flushing head for use at any other location in the system was fitted with $25 \mathrm{~mm}$ air and water fittings. Detailed operational procedures were in place for a blocked line scenario with predetermined set points for alarms and auto dump functions.

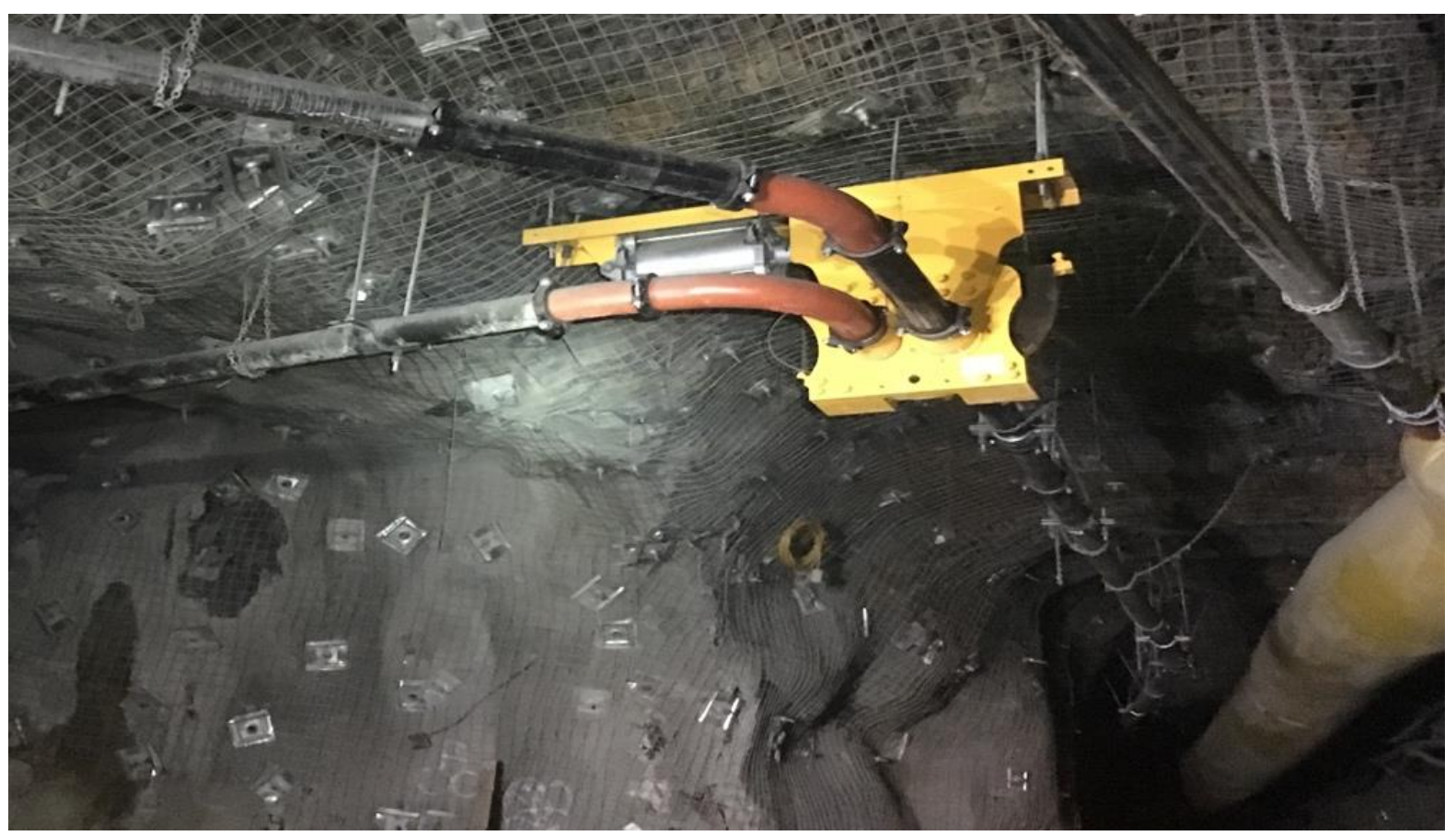

Figure 2 QD2 installed in the backs at the 9040L

\section{$4 \quad$ The blockage}

On 6 July 2018 at $12.40 \mathrm{am}$, the lowest installed pressure sensors on the QD2s at 8790L and 9040L spiked from a normal operating pressure of 0.5-8 MPa within four minutes, triggering automatic dump sequences. The QD2 at 9430L (the base of the borehole) spiked from a normal operating pressure of about 3-10 MPa in a matter of seconds, also triggering an automatic dump sequence. Figure 3 provides a graphical representation of the pressure versus time recorded by the various pressure sensors during the event. It can be observed that the pressure sharply rises in all three locations in a short period of time.

The pressure spikes reached the auto dump sequence set point of $8 \mathrm{MPa}$ nearly simultaneously, initiating the paste plant to commence an immediate shutdown and all three QD2s to divert to their respective dump positions. This action allowed the paste to discharge from the reticulation system in seconds, dropping the pressure back to zero.

Once the line pressures had dropped below $8 \mathrm{MPa}$, the paste plant operator initiated the 'blocked line' protocol. Starting in a top-down manner, the paste plant operator flushed each section of the reticulation clean with $5 \mathrm{~m}^{3}$ of water, followed by air, ensuring each section was fully recovered before closing the QD2 and flushing to the next QD2 location. This process took about 20 minutes to complete and the reticulation was confirmed clear from the surface down to the last QD2 location (8790L). Essentially, three-quarters of the $1,850 \mathrm{~m}$ reticulation system, including an AUD 2 million borehole, were saved in a matter of minutes safely, efficiently and effectively. 


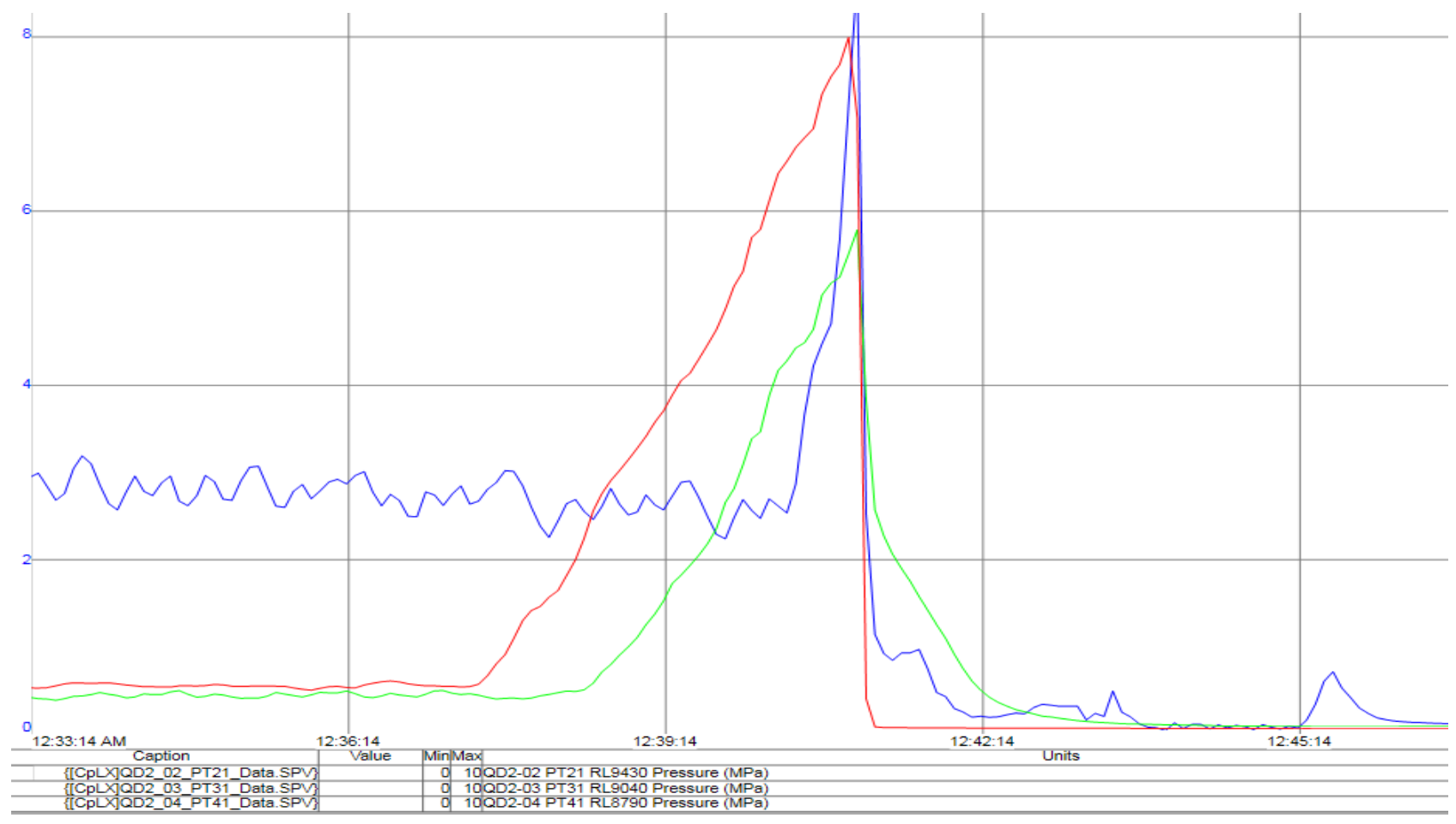

Figure 3 Pressure versus time graph during the blockage (MPa, 8790-Red, 9040-Green 9430-Blue)

Once the reticulation had been cleared and flushed clean to $8790 \mathrm{~L}$, the paste plant operator began flushing the remainder of the system, which had no QD2 or instrumentation installed at that stage. After attempting to flush to the fill location on $8670 \mathrm{~L}$ for 40 minutes without movement, the balance of the reticulation was now considered to be blocked.

Once the lower section of the reticulation was considered blocked, the underground paste crew and paste foreman commenced a manual unblocking sequence and began using the mobile flushing head to flush the reticulation system. An attempt was made to flush the system from $8670 \mathrm{~L}$ to $8700 \mathrm{~L}$ with a mobile flushing head (Figure 4) with no success. The mobile flushing head was attached to the reticulation at $8700 \mathrm{~L}$ and that level was successfully flushed. An attempt was then made to flush from $8700 \mathrm{~L}$ to the fill point at $8670 \mathrm{~L}$ with no success. It is worth noting that at this stage five hours had passed, and the binder content was relatively high, resulting in the paste curing inside the pipe.

Moving down to $8670 \mathrm{~L}$, the elbow joining the 8670 to 8700 inter-level boreholes was removed, which allowed the paste to flow out with the assistance of gravity. The mobile flushing head was only able to flush $20 \mathrm{~m}$ sections as the paste was at an almost solid consistency by this time.

Underground firing was postponed as per the procedure and the operators were hot seated underground so the clearing of pipes could continue into the next shift without losing any more time. The pipes were broken into 6-12 $\mathrm{m}$ lengths for flushing with the mobile flushing head. The paste had cured very close to solid by the end of the day shift; however, the underground crew was able to fully clear the reticulation system.

Note: The post-incident investigation found that an unlined inter-level borehole failure was the cause of the blockage. The inter-level borehole between $8700 \mathrm{~L}$ and $8730 \mathrm{~L}$ had to be redrilled as a result. The whole paste fill system was operational again within three days. 


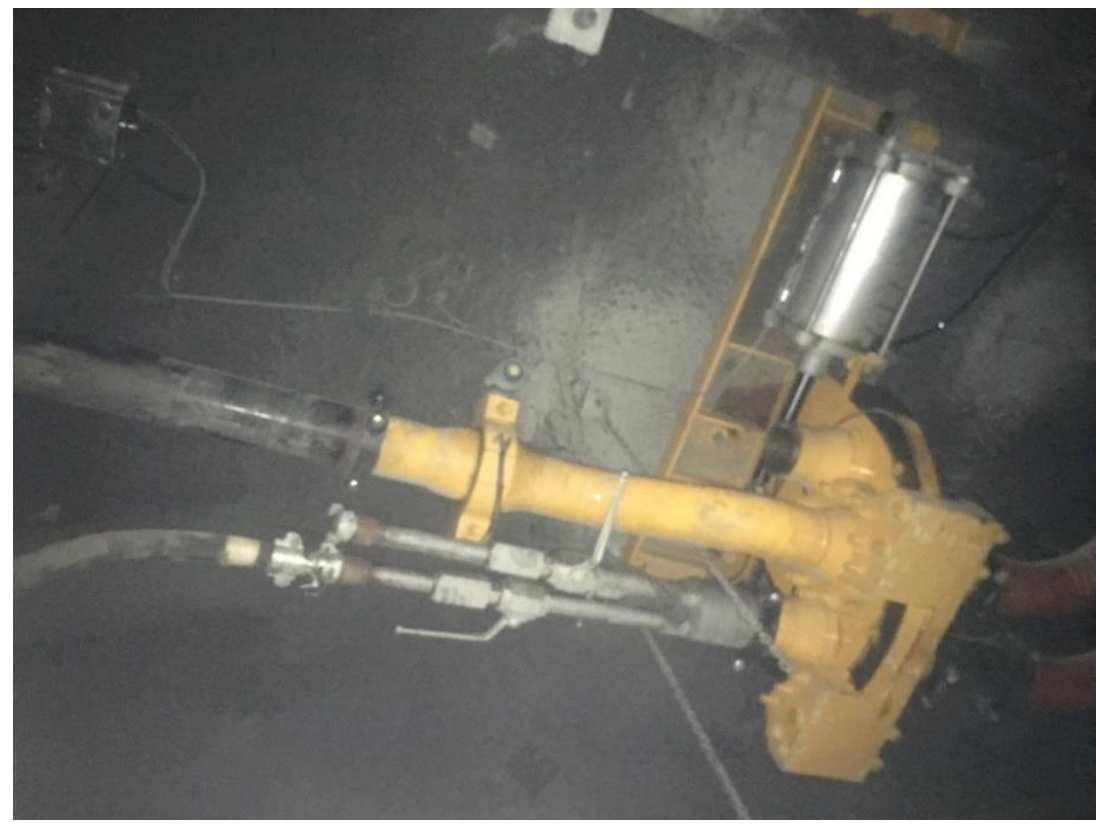

Figure 4 QD2 with flushing head attached

The flushing heads shown in Figure 5 were custom designed for the project. The larger flushing head is connected to each QD2 to flush the downstream line when the QD2 diverts into dump position, while the smaller flushing head is a mobile unit that can be taken to an intermediate location. The units are designed for $7 \mathrm{MPa}$ pressures while the connecting end is grooved to suit the reticulation coupling to attach effectively to underground pipe work.

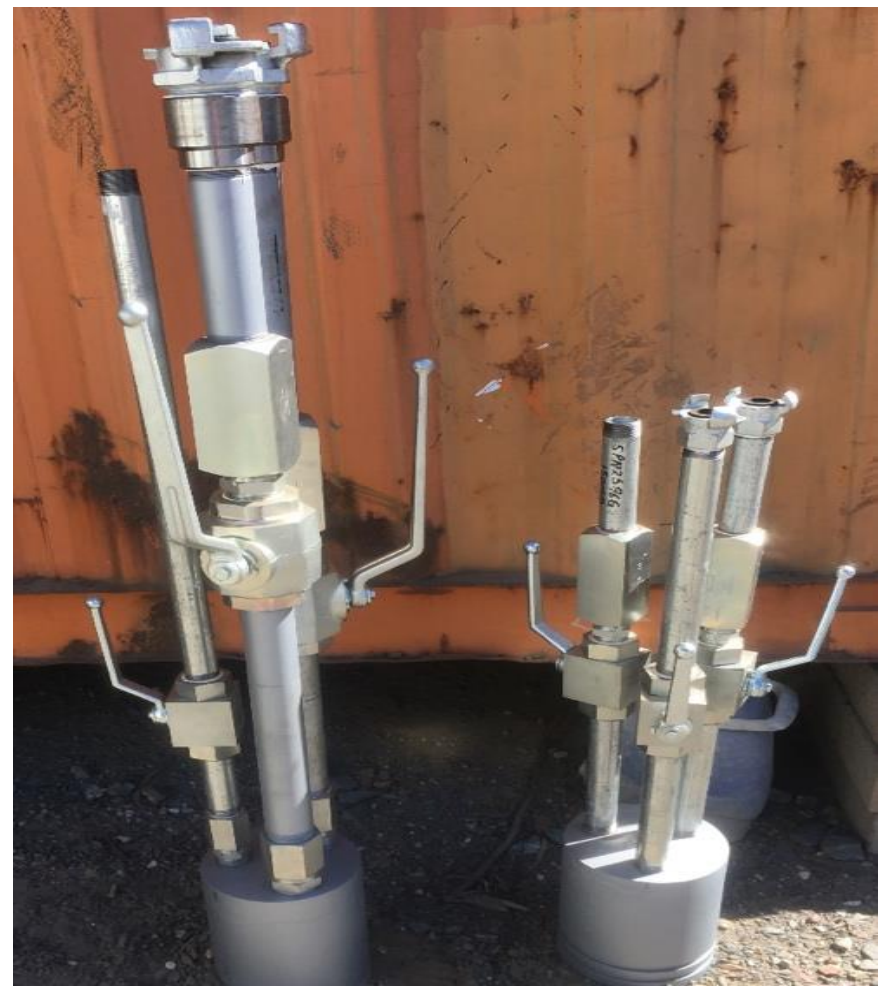

Figure 5 Flushing head units for attaching to QD2, Quattro Changeover Spools and underground paste pipes 


\section{Conclusion}

Once an investigation was completed, several issues were identified:

- Excessive loss of reaction time was experienced during the manual unblocking sequence due to limited tooling, and the time for tramming integrated tool carriers from location to location over large distances and searching for other equipment such as work baskets. The splitting of resources to work above and below the failed borehole also contributed to the delays. The ability to automatically unblock the upper levels using the QD2s mitigated these delays to a great extent.

- The lower inter-level boreholes are 30-40 m in length and it became apparent that with the progressive curing of the paste, its flow ability became almost non-existent. Thus, the only way to dump or evacuate paste from the lower inter-level boreholes was for personnel to manually open the pipe at the couplings. This was not ideal. Although the paste line was isolated, the inter-level borehole still potentially had stored energy from the available hydraulic head of the paste of around $40 \mathrm{~m}$. The release of stored energy along with 'springing' of the pipe work (a movement of the pipes which is common in many paste fill reticulation system) creates a less than desirable environment for personnel to be working in.

- It had been proven onsite prior to the blockage that if a flushing head is used while the paste is still relatively fluid (up to four hours), a single flushing head is capable of flushing multiple levels clean at a time. With the slower manual unblocking process and associated delays, the paste backfill in the lower sections experienced some curing, leading to reduced efficiency of the flushing heads that were ultimately limited to flushing $20 \mathrm{~m}$ sections.

The ability to isolate the QD2s underground to allow personnel to start working on the blocked reticulation proved to be invaluable. If this were not possible, it would have required a two-hour round trip every time the paste reticulation system needed to be isolated on the surface. The blockage and subsequent reaction to the blockage was considered a success.

To rectify highlighted risks to the paste fill operation and personnel, extensive upgrades to the infrastructure in the lower levels and the blockage reaction plan or 'emergency blockage procedure' have taken place.

A change of management plan was conducted, resulting in the decision to install paste flushing stations (PFS) at strategic locations of high value or risk. This PFS consists of a permanently installed QD2 or a Quattro Changeover Spool (QCS) as shown in Figure 6, with a flushing head plumbed into the mine's water and air DN63 services. This equipment is accessed from an aluminium work platform (Figure 7) on a concrete pad $2.5 \times 3.5 \mathrm{~m}$, enabling personnel to work on the reticulation safely. This also negates the need for an integrated tool carrier to be used, saving valuable time at the most critical juncture of a blockage. The platform is costeffective, lightweight enough to be moved by hand if necessary, does not need personnel to have any specific training to assemble, and has a working height of up to $5.3 \mathrm{~m}$. 


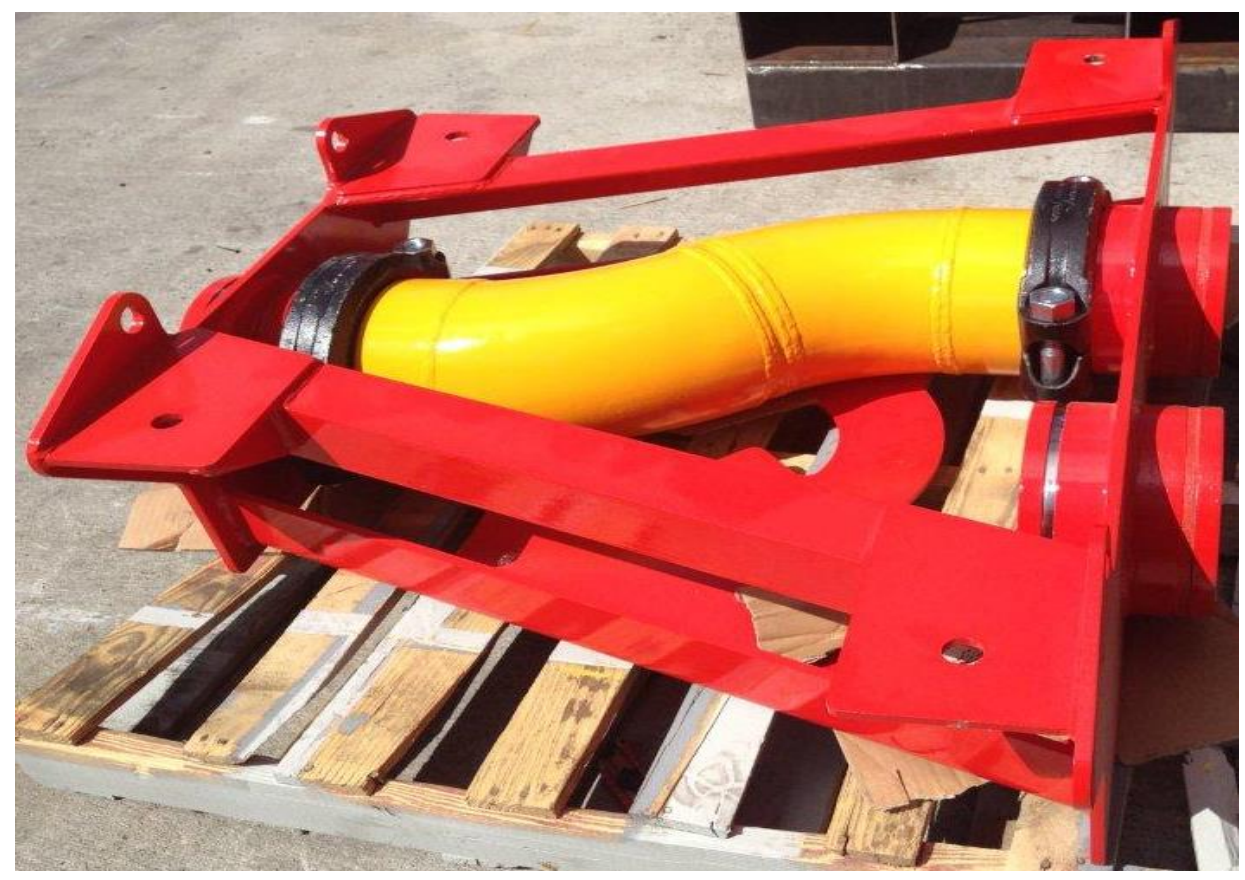

Figure 6 QCS - manual changeover spool

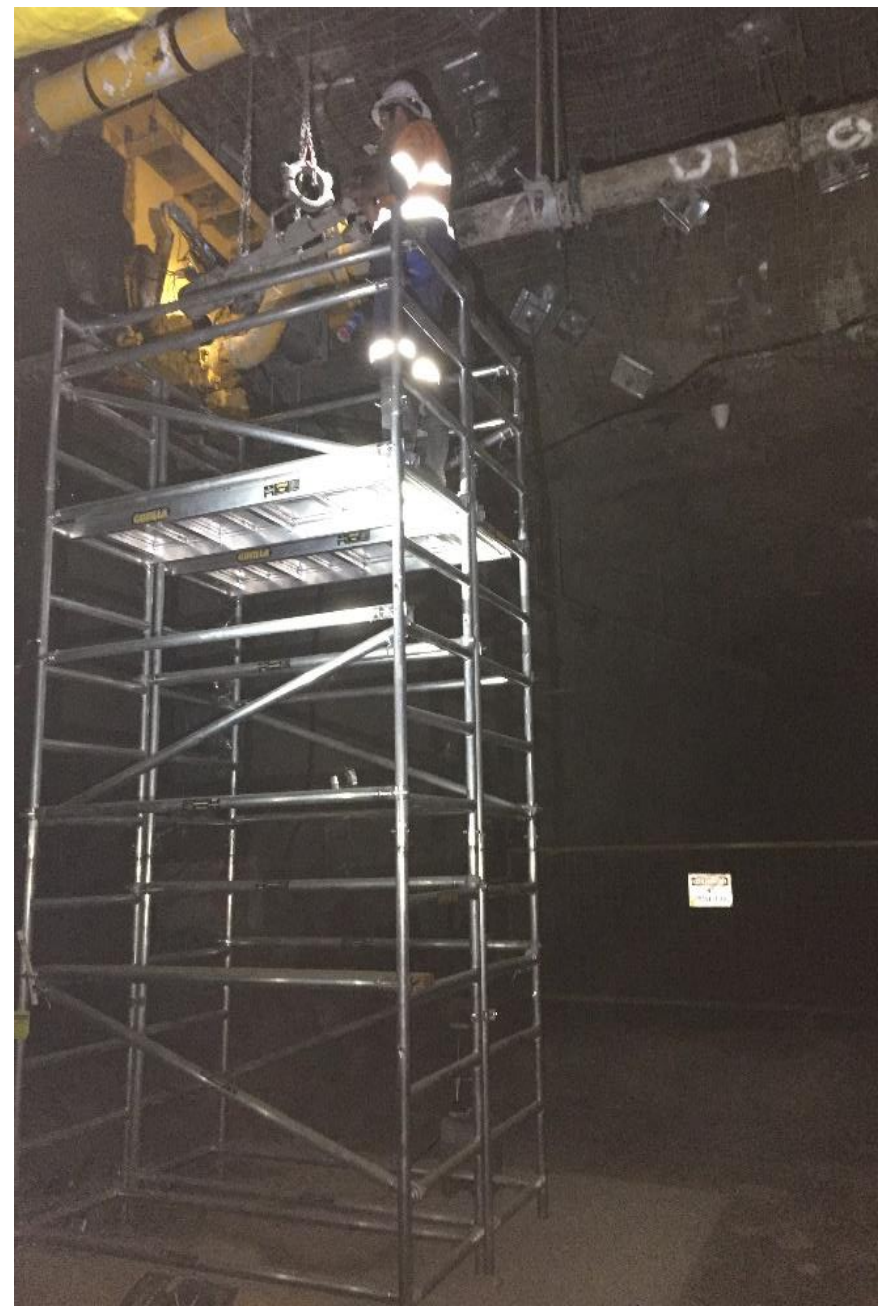

Figure 7 Shows the platform and concrete pad used as part of a paste flushing station 
The benefits of the PFS means that any stored energy can be bled or released safely via the installed QCS/QD2 and has the advantage of having the permanent flushing head attached for immediate use. Adding a work platform to this installation, along with the ability for personnel to isolate the QD2 from the control box (Figure 8) on ground level, means an extremely fast reaction time to any line blockage.

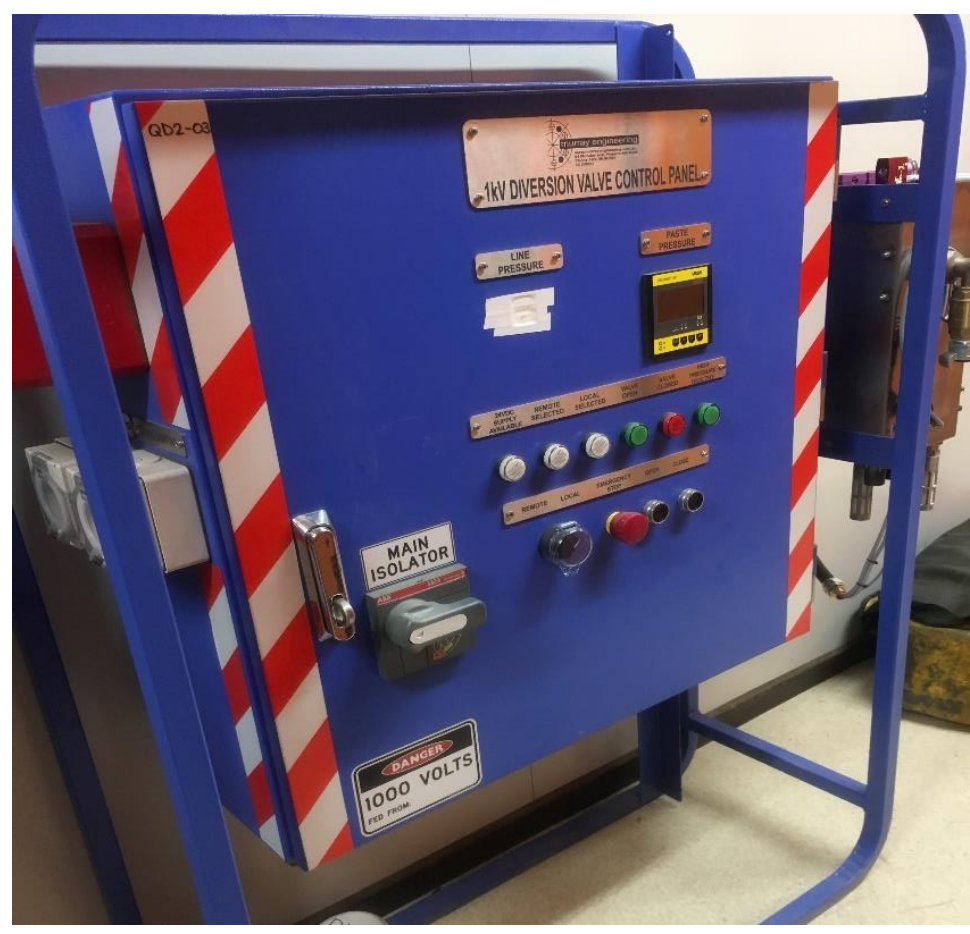

Figure 8 QD2 underground control panel with UPS, closed communications circuit and air receiver

To complement the six PFSs installed so far, two mobile flushing heads and two locked toolboxes with all the equipment needed are located underground in the event of a future blocked line emergency.

A well-planned emergency procedure to help overcome a stressful situation (blocked paste line) with high potential losses is essential. It is also critical that the procedures define the paste plant operator's actions at critical junctions early in the blockage. An isolation protocol with multiple crews working at the same time and a clear action plan for clearing the blockage is also essential. The procedure has subsequently been refined and updated to make it possible for supervisors, who may not have a lot of exposure to the day-to-day operations of paste fill, to deploy the procedure and direct multiple crews who themselves may not work directly within the paste fill department. This allows 24-hour coverage of the paste fill operation.

\section{Acknowledgement}

The author thanks Quattro Project Engineering for the continual support, innovative products line and for always looking at problems as a potential opportunity to find and implement a solution; and Cobar Management Pty Ltd CSA Mine NSW Australia's management team for always being ready to support and proactively pursue practical solutions throughout the project. 